\title{
On the Reference Pole for Earth Orientation and UT1
}

\author{
P. M. Mathews \\ Department of Theoretical Physics, University of Madras, Chennai 600 \\ 025, India
}

\author{
T. A. Herring \\ Department of Earth, Atmospheric, and Planetary Sciences, M. I. T., \\ Cambridge, MA 02139
}

\begin{abstract}
.
We show how the study of variations in orientation of a terrestrial reference system (TRS) in space may be done directly in terms of the motion of the pole of the TRS and rotation around it, and how a separation of these variations into low frequency and high frequency (retrograde and prograde diurnal, semidiurnal, $\cdots$ ) bands enables one to characterize and model variations belonging to the various bands and to estimate them simultaneously from observational data by a uniform procedure. Introduction of the Celestial Ephemeris Pole (CEP) or other Celestial Intermediate Pole (IP) is not only unnecessary, but also gives rise to needless debate as to whether variations due to particular causes are to be included in the celestial motion of the IP or in its terrestrial motion, and leaves the question of estimation of high-frequency signals in either frame unresolved. In regard to UT1, we point out that the "correction terms" through which the concept of the nonrotating origin is implemented emerge naturally from fundamental kinematical relations, and use this observation to identify the correction terms to be employed when the Earth orientation parameters are defined in relation to the pole of the TRS rather than an IP.
\end{abstract}

\section{Introduction}

It has been a widely held belief that estimation of the high-frequency part (with periods below 2 days) of the variations in Earth orientation cannot be done by analysis of VLBI data gathered routinely in one-day sessions at intervals of several (typically 5 to 7 ) days. The notion of a Celestial Ephemeris Pole (CEP) which has no high-frequency components to its motion in terrestrial as well as celestial reference frames is a counterpart of this belief on the conceptual front, though, a causal connection between the two is hard to pinpoint. Both of these ideas are reflected in the traditional (and current) procedure for analysis of VLBI data, which decomposes Earth orientation variations into separate motions of the CEP in the celestial and terrestrial reference frames, with no high-frequency content in either, and a nearly diurnal rotation around this pole. The investment 
made over a couple of decades in algorithms and software which implement this procedure is one of the potent factors which deters any significant move away from this pole, and understandably so.

Yet, higher frequencies are present in Earth rotation variations, and their amplitudes can, in fact, be estimated with considerable precision from existing VLBI data sets, notwithstanding the belief mentioned at the outset: Herring and Dong (1994) showed how when they estimated the amplitudes of ocean-tideinduced variations having diurnal and semidurnal frequencies in the terrestrial frame. Recent works on rigid Earth nutations have shown that diurnal and semidiurnal frequencies occur also in the motions of the Earth's axis in space. The concept of a slow-moving CEP has thus lost its relevance.

The main question addressed here is: do we need a replacement for the CEP? To put it differently, would an Intermediate Reference Pole help us to accomplish something that cannot be accomplished without it? We believe the answer to be in the negative: an intermediate pole is redundant. We outline our reasoning here.

Stated briefly: The information on Earth orientation provided by groundbased observations of celestial objects relates to the orientation of an Earthfixed reference frame, and in particular, that of its third axis. Even when Earth orientation parameters (EOP) are defined in relation to the CEP, their variations have to be inferred from the observable quantities, which relate only to the motion of the TRS in space. Even with the present methods of VLBI data analysis, they are, in actual fact, obtained in this manner, as will be made manifest below. The seeming role of the CEP merely masks this basic fact.

The definition of the axial rotation of the Earth, and hence that of UT1, is tied to the choice of the axis about which the rotation is to be defined. The concept of the nonrotating origin (Guinot, 1979) may be implemented with any chosen axis. We draw attention here to the fact that the small "corrections" (Capitaine et al., 1986) to be applied to obtain UT1 from the finite angle of rotation about that axis - defined in the decomposition of Earth rotation into motions of the pole and axial rotation around the pole - follow directly from the kinematical relations connecting the Earth rotation parameters (spin rate variation, and wobble) to the Earth orientation parameters.

\section{Transformation from CRS to TRS via an intermediate pole}

The transformation from a celestial reference system (CRS) to a terrestrial one (TRS) via an intermediate pole (IP) is

$$
T=W R S,
$$

where $S$ takes the pole of the CRS into the IP, $R$ represents rotation around the IP through a finite angle $\chi$, and $W^{-1}$ takes the pole of the TRS into the IP.

$$
\begin{gathered}
R=\left(\begin{array}{ccc}
\cos \chi & \sin \chi & 0 \\
-\sin \chi & \cos \chi & 0 \\
0 & 0 & 1
\end{array}\right), \\
S=\left(\begin{array}{ccc}
1 & 0 & -X \\
0 & 1 & -Y \\
X & Y & 1
\end{array}\right)-\frac{1}{1+\left(1-X^{2}+Y^{2}\right)^{1 / 2}}\left(\begin{array}{ccc}
X^{2} & X Y & 0 \\
X Y & Y^{2} & 0 \\
0 & 0 & X^{2}+Y^{2}
\end{array}\right),
\end{gathered}
$$


where $(X, Y)$ denote the coordinates of the IP in the CRS, and $\chi$ is the finite angle of rotation around the IP. $W$ has the same form as $S$ except for the replacements $X \rightarrow-\xi, Y \rightarrow-\eta$ where $(\xi, \eta)$ are the coordinates of the IP in the TRS, related to the familiar polar motion parameters by

$$
\xi=x_{p}, \eta=-y_{p}
$$

(Only the part of $W$ that is linear in $(\xi, \eta)$ is retained in VLBI analysis at present, the higher order part being deemed negligible.) $S$ represents precession and nutation, and $W$, the "polar motion". $X, Y, \xi, \eta$, and $\chi$ constitute the Earth orientation parameters. The definition of the CEP envisages that the spectra of the first four of these contain only low frequency components. For instance,

$$
\begin{gathered}
X=\sum_{\tau}\left[A_{\tau} \sin \theta_{\tau}+A_{\tau}^{\prime} \cos \theta_{\tau}\right], \quad Y=\sum_{\tau}\left[B_{\tau} \cos \theta_{\tau}+B_{\tau}^{\prime} \sin \theta_{\tau}\right], \\
\theta_{\tau}=\tau \Omega_{0} t+\alpha_{\tau},
\end{gathered}
$$

where $\Omega_{0}$ is the mean sidereal rotation rate of the Earth, and the sum is over spectral components with $0<\tau<(1 / 2)$.

In current practice, the expression used for $S$ is in terms of the nutations in longitude and obliquity, $\Delta \psi$ and $\Delta \epsilon$, not the Cartesian $(X, Y)$; the transformation between these may be found in Capitaine (1990).

One sees readily that the coordinates $\left(X_{T}, Y_{T}\right)$ of the terrestial pole (pole of the TRS) in the CRS are just the $(1,3)$ and $(2,3)$ elements of the matrix $T^{-1}$, and hence that

$$
X_{T} \approx X-\xi \cos \chi+\eta \sin \chi+, \quad Y_{T}=Y-\xi \sin \chi-\eta \cos \chi,
$$

to the first order in $(X, Y)$ and $(\xi, \eta)$. The largest of the higher order terms in $X_{T}$ is $X F$, and that in $Y$ is $Y F$, where

$$
F=[X(\xi \cos \chi-\eta \sin \chi)+Y(\xi \sin \chi+\eta \cos \chi)] .
$$

The magnitude of $F$ is quite small. Precession at the rate $p \approx 50^{\prime \prime}$ per year contributes $100 p \sin \epsilon_{0} \approx 2000^{\prime \prime} \approx 0.01$ radian to $X$ over a century while $\xi$ and $\eta$ are under $0.4 \approx 2 \times 10^{-6}$ radian. So $F$ does not exceed $2 \times 10^{-8}$ over a century, the corresponding correction to $X_{T}$ of (3) would be less than $40 \mu$ as over a century. The periodic part of the correction is under $0.2 \mu$ as for both $X$ and $Y$. The linear approximation (3) is therefore sufficient for present purposes. The expression for the complex combination $\tilde{X}_{T} \equiv\left(X_{T}+i Y_{T}\right)$ is more revealing than eqs. (3) as they stand:

$$
\tilde{X}_{T}=\tilde{X}-\tilde{\xi} e^{i \chi}, \quad(\tilde{X}=X+i Y, \tilde{\xi}=\xi+i \eta) .
$$

The expansions (2) for $X$ and $Y$ lead to

$$
\tilde{X}=\sum_{\tau} C_{\tau} e^{i \tau \Omega_{0} t}
$$

where the sum is now over both positive and negative (prograde and retrograde) frequencies in the range $\left(-\Omega_{0} / 2\right)$ to $\Omega_{0} / 2$. The expansion of $\tilde{\xi}$ is similar. 
The nature of the terms containing the polar motion variables in eqs. (3) or (5) deserves special attention: with $\xi$ and $\eta$ taken (as in routine VLBI analysis) to contain no high frequency components, these terms represent purely prograde diurnal components of the motion of the terrestrial pole in space if we leave aside for the moment the extremely small terms in other frequency bands (to be considered later) that arise from the diurnal and semidiurnal UT1 variations contained in $\chi$. There is no place within this scheme for semidiurnal or retrograde diurnal signals in $X$ and $Y$ due, for instance, to ocean or atmospheric tides. Herring and Dong (1994) overcame this limitation by a scheme which is equivalent to replacing the slowly varying $\tilde{\xi}$ in (5) by

$$
\tilde{\xi}_{0}+\sum \tilde{\xi}_{n} e^{i n \Omega_{0} t}
$$

wherein $\tilde{\xi}_{n}$ for every $n$ contains only low frequencies (between $\left(-\Omega_{0} / 2\right)$ and $\left(\Omega_{0} / 2\right)$.) When this is introduced in $(5), \tilde{\xi}_{0}$ takes the place of $\tilde{\xi}$, while the term for any $n \neq 0$ represents signals within a band of width $\Omega_{0}$ centered at the frequency $(n+1) \Omega_{0}$ in space.

\section{Is an Intermediate Pole necessary?}

Long usage of the factorization (1) of the transformation between the CRS and the TRS, and the accompanying identification of the polar motion parameters in $W$ with the coordinates of an Intermediate Pole (hitherto the CEP) has led to a universal and unquestioning belief that an Intermediate Pole is essential to make it possible to estimate both the precession-nutation and the geophysically induced rotation variations (such as the Chandler wobble) as low frequency signals from VLBI data.

Remember, however, that the VLBI observables depend on Earth orientation only through three independent parameters, say $X_{T}, Y_{T}$, and the angle of rotation $\chi_{T}$ around the terrestrial pole. For the estimation of $\xi$ and $\eta$ from the data, the manner in which they enter into the prograde diurnal part of $\left(X_{T}, Y_{T}\right)$ is all that is relevant, not any interpretation as coordinates of some intermediate pole. Independently of this line of reasoning, one of us (Mathews, 1999) had proposed a scheme for the modeling and estimation of signals in any frequency band $(n-1 / 2) \Omega_{0}$ to $(n+1 / 2) \Omega_{0}$ of interest in the CRS or the TRS. If the terrestrial pole itself (rather than some Intemediate Pole) is chosen as the reference pole, then the scheme reduces to (i) expanding $\left(X_{T}, Y_{T}\right)$ as

$$
\begin{aligned}
& X_{T}(t)=X_{0}(t)+\sum\left[X_{n}(t) \cos \left(n \Omega_{0} t\right)-Y_{n}(t) \sin \left(n \Omega_{0} t\right)\right], \\
& Y_{T}(t)=Y_{0}(t)+\sum\left[X_{n}(t) \sin \left(n \Omega_{0} t\right)+Y_{n}(t) \cos \left(n \Omega_{0} t\right)\right],
\end{aligned}
$$

with the spectrum of every $X_{n}(t)$ and $Y_{n}(t)$ confined to low frequencies (0 to $\Omega_{0} / 2$ ), and (ii) estimating as many pairs $\left(X_{n}(t), Y_{n}(t)\right)$ as are desired, subject to limitations imposed by the quality of the data. The terms corresponding to a given $n \neq 0$ in $X_{T}(t)$ and $Y_{T}(t)$ would contain frequencies between $(n-1 / 2) \Omega_{0}$ and $(n+1 / 2) \Omega_{0}$ in the CRS, prograde (retrograde) for positive (negative) $n$; 
and $\left(X_{0}(t), Y_{0}(t)\right)$ represents the low frequency band $\left(-1 / 2 \Omega_{0}\right.$ to $\left.1 / 2 \Omega_{0}\right)$ in the CRS. This is manifest from the complex version of (8):

$$
\tilde{X}_{T}(t)=\tilde{X}_{0}(t)+\sum \tilde{X}_{n}(t) e^{i n \Omega_{0} t},
$$

the spectral expansion of $\tilde{X}_{n}(t)$ being

$$
\tilde{X}_{n}(t)=\sum_{-1 / 2<\tau<1 / 2} \tilde{X}_{n, \tau} e^{i \tau \Omega_{0} t}
$$

It is now evident that if (8) were truncated by dropping all but the $n=1$ term in the sum, the result would be just the same as (3) with only one difference: the replacement of $\chi$ in (3) by $\Omega_{0} t$ in (8). It may be verified that the contribution to $X_{T}$ or $Y_{T}$ in (3) from the largest of the diurnal and semidiurnal terms in the difference $\left(\chi-\Omega_{0} t\right)$ is under $10^{-6}$ mas and hence entirely negligible. The low frequency part of $\left(\chi-\Omega_{0} t\right)$ would contribute to the low frequency part of $\left(X_{T}, X_{T}\right)$. The difference between $\left(X_{0}, Y_{0}\right)$ and $(X, Y)$ of eq. $(3)$ that arises therefrom is well under the $\mu$ as level, except for the contribution from the 18.6 year term in $\chi$ which is still under $5 \mu$ as. Except for this tiny difference, $\left(X_{0}, Y_{0}\right)$ of $(8)$ is just the low frequency $(X, Y)$ of $(3)$. More importantly, $\left(X_{1}, Y_{1}\right)$ is seen to be just the same as $(\xi, \eta)$, though the former is not identified with the terrestrial motion of any Intermediate Pole: no such pole was employed in the derivation of $(8)$.

We may therefore draw from the correspondence above the important conclusion that the invocation of an Intermediate Pole in the traditional decomposition of the transformation $T$ is not of any real relevance to the definition of the Earth orientation parameters, and that one may work just as well with the terrestrial pole itself, and the parameters $\left(X_{0}, Y_{0}\right),\left(X_{1}, Y_{1}\right)$, and $\chi_{T}$ associated with it.

A further important fact is that (8) already contains additional terms representing frequency bands other than the two present in (3): $n=2,3, \cdots$ for prograde semidiurnal, terdiurnal, $\cdots$ bands of frequencies in space, and $n=$ $-1,-2, \cdots$ for retrograde diurnal, semidiurnal, $\cdots$ ones. The signals in one or more of these bands (due, for instance, to ocean or atmospheric tides) can be found simply by estimating the corresponding low frequency functions $\left(X_{n}, Y_{n}\right)$ along with those for $n=0$ and 1 and $\chi_{T}$. This procedure is essentially equivalent to, but more direct than, that pioneered by Herring and Dong (1994); it has been found successful in tests done recently by Ch. Bizouard, A.-M. Gontier, and N. Capitaine (private communication, 2000).

Yet another fact of some importance is that, there being no intermediate pole, there is no scope for the oft-debated question as to whether signals in one frequeny band or the other are to be visualized as and estimated in the CRS or in the TRS, or for the related question as to whether signals arising from some particular physical cause (e.g., the diurnal and semidiurnal nutations produced by external gravitation) should be modeled in the CRS or the TRS. All terms in expressions (8) and (9) are, unambiguously, parts of the motion of the terrestrial pole in the CRS. 


\section{Modeling and estimation}

Modeling is done by using expressions of the form (2) for each of the $X_{n}(t)$ and $Y_{n}(t)$, with values derived from theory for the coefficients $A_{n, \tau}, A_{n, \tau}^{\prime}, B_{n, \tau}, B_{n, \tau}^{\prime}$. For nutations caused by external gravitating bodies, one has, besides the main terms corresponding to $n=0$, also the recently discovered terms belonging to $n=1$ and 2. For variations of geophysical origin, signals having terrestrial frequencies $\sigma \Omega_{0}$ with $\sigma$ in the range $(r-1 / 2)<\sigma<(r+1 / 2)$ are represented by the term with $\mathrm{n}=(\mathrm{r}+1)$ in (9). In particular, one has a term $\tilde{X}_{r+1, \tau} e^{i(r+1) \Omega_{0} t}$ corresponding to a wobble of frequency $\sigma \Omega_{0}$ and amplitude $\tilde{m}(\sigma),(\sigma=r+\tau)$ :

$$
\tilde{X}_{r+1, \tau}=-\frac{\tilde{m}(\sigma)}{1+\sigma}, \quad(\sigma=r+\tau) .
$$

Modeling of such a term can be done if a satisfactory theoretical model is available for the computation of $\tilde{m}(\sigma)$. A wobble $\tilde{m}(\sigma)$ due to a prograde diurnal ocean tide, for example, would contribute a term $\tilde{X}_{2, \tau} e^{i(2+\tau) \Omega_{0} t}$ in the semidiurnal band to $\tilde{X}_{T}(t)$; semidiurnal nutation terms excited by external gravitation also appear in the same band.

\section{Kinematic relations}

The components $\Omega_{0} m_{1}, \Omega_{0} m_{2}$, and $\Omega_{0}\left(1+m_{3}\right)$ of the Earth's angular velocity vector $\Omega$ in the TRS are the off-diagonal elements of the antisymmetric matrix $M \equiv \dot{T}^{-1} T$, where the transformation matrix $T$ is just $R S$ in the absence of any Intermediate Pole. Evaluation of $M$ leads to the kinematic relations

$$
\begin{gathered}
\Omega_{0} m_{1}=L_{23} \equiv(\dot{X}+X \Delta) \sin \chi-(\dot{Y}+Y \Delta) \cos \chi, \\
\Omega_{0} m_{2}=L_{31} \equiv(\dot{X}+X \Delta) \cos \chi+(\dot{Y}+Y \Delta) \sin \chi \\
\quad \Omega_{0}\left(1+m_{3}\right)=L_{12} \equiv \Omega+\Delta_{3}, \\
\Delta \equiv X \dot{X}+Y \dot{Y}, \quad \Omega=\dot{\chi}, \quad \Delta_{3}=(1 / 2)(Y \dot{X}-X \dot{Y}) .
\end{gathered}
$$

Eq. (10) is the frequency domain version of the equation $\Omega_{0} \tilde{m}=i \dot{X} e^{-i \Omega_{0} t}$ that is equivalent to the linearized form $\left(\Delta \rightarrow 0, \chi \rightarrow \Omega_{0} t\right)$ of the pair of eqs. (11a), (11b); this approximation is more than adequate for the purpose for which the use of $(10)$ was envisaged.

\section{Definition of UT1}

UT1 is intended to be proportional to the angle of rotation of the Earth in space. It would seem eminently reasonable to require that the reference pole around which the rotation is to be defined be a physically meaningful one, such as the pole of the TRS or the instantaneous rotation pole - not some "Intermediate Pole" (the CEP or some modified version of it) that, strictly speaking, has no real physical existence. It is pertinent to point out in this context that UT1 variations of geophysical origin (due to zonal tidal deformations) must 
necessarily be identified with variations in rotation around the pole of the TRS because the third axis of the TRS is the reference axis for the definition of the zonal tides. The choice of a different reference pole for the fundamental definition of UT1 would then seem incongruous.

When the Earth orientation parameters are defined in relation to an IP, the third of the kinematical relations has the general form

$\Omega_{0}\left(1+m_{3}\right)=L_{12}-(1 / 2)\left(y_{p} \dot{x}_{p}-x_{p} \dot{y}_{p}\right)-\left[(1 / 2) L_{12}\left(x_{p}^{2}+y_{p}^{2}\right)-\left(L_{23} x_{p}-L_{31} y_{p}\right)\right]$,

instead of (11c), where the $L_{i j}$ are defined as in eqs. (11). Recalling that $L_{12}=d \chi / d t+\Delta_{3}$, we find the angle of rotation around the pole of the TRS to be

$$
\int \Omega_{0}\left(1+m_{3}\right)=\chi+(1 / 2) \int(Y \dot{X}-X \dot{Y}) d t-(1 / 2) \int\left(y_{p} \dot{x}_{p}-x_{p} \dot{y}_{p}\right) d t+\cdots,
$$

wherein the dots represent the integral of the square-bracketed part of (13), and we have used the expression for $\Delta_{3}$ from (12). One recognizes the terms following $\chi$ in (14) to be of just the same forms as the quantities $s$ and $s^{\prime}$ involved in the implementation of the nonrotating origin by Capitaine (1990); $\chi$ with these terms added constitutes the basis for her definition of UT1, when the pole of reference is the CEP or a redefined IP. If the pole of reference were the pole of the TRS itself, the replacements $\chi \rightarrow \chi_{T},(X, Y) \rightarrow\left(X_{0}, Y_{0}\right)$ and $\left(x_{p},-y_{p}\right) \rightarrow$ $\left(X_{1}, Y_{1}\right)$ would lead to the corresponding quantity, $\chi_{T}+(1 / 2) \int\left(Y_{0} \dot{X}_{0}-X_{0} \dot{Y}_{0}\right) d t+$ $(1 / 2) \int\left(Y_{1} \dot{X}_{1}-X_{1} \dot{Y}_{1}\right) d t$ as the appropriate basis for the definition of UT1.

It would seem therefore that there is no need for an Intermediate Pole for the definition of UT1 either.

\section{References}

Capitaine, N., 1990, "The celestial pole coordinates," Celest. Mech. Dyn. Astron., 48, 127-143.

Capitaine, N., Guinot, B., and Souchay, J., 1986, "A nonrotating origin on the instantaneous equator: Definition, properties and use," Celest. Mech., 39, 283-307.

Guinot, B., 1979, "Basic problems in the kinematics of the rotation of the Earth," in Time and the Earth's rotation, D. D. McCarthy and J. D. Pilkington (eds), D. Reidel Publishing Company, 7-18.

Herring, T. A. and Dong, D., 1994, "Measurement of diurnal and semidiurnal rotational variations and tidal parameters of the Earth," J. Geophys. Res., 99, 18051-18071.

Mathews, P. M., 1999, "Proposal for new conceptual definition of the celestial ephemeris pole and procedure for realization of the pole," Proc. Journées Systèmes de Référence Spatio-temporels 1998, Observatoire de Paris, 161163. 